\title{
Research of Detection Algorithm about Rectangular Degree of Metal Parts Based on Halcon
}

\author{
Xin Liu ${ }^{1, a, \star}$, Ying Mu ${ }^{1, b}$, Hongbin Wang ${ }^{1, c}$ \\ Department of Electronic and Information Engineering, Lanzhou Institute of Technology Lanzhou, \\ 730050, China \\ afallingstar0505@163.com, b33631643@qq.com, c516507644@qq.com
}

Keywords: Halcon; Rectangular Degree; Template Matching Based On Edge; Machine Vision.

\begin{abstract}
A new approach of the rectangular degree detection of the metal parts is proposed. Our design is based on Halcon, a machine vision software, which includes key processing steps shuch as the edge contour extraction, image matching, and defect detection and so on. First, the edge is extracted according to the characteristics of the metal parts automatically. Then, the template matching based on edge is realized to measure the rectangular degree of metal parts. At last, the algorithm to detect the defects is used. The results of experiment show the improved algorithm can automatically detect the rectangular degree of metal parts, so it has a certain validity and feasibility.
\end{abstract}

\section{Introduction}

The metal part are extensively used in industrial domains such as automobile, plane and building, etc. However, in the cutting process, the fabrication process causes cracks, disqualified parameter indices, and scratches, etc. to directly influence the abrasion resistance, corrosion resistance and limit of fatigue of machine element. With higher requirements put forward on defects detection for metal part by people, to research a method to rapidly survey and analyze parameters of metal part is of important scientific significance ${ }^{[1]}$. The time and efforts consuming traditional artificial detection method is hard to meet production requirement. In recent years, people introduced machine vision into quality detection of metal part, with advantages of non-contacting, quick speed, and strong resistance to jamming, this method improves efficiency and accuracy of measurement, and becomes a focus of attention.

The paper combines Halcon machine vision software to suggest a design proposal for detection of rectangularity of metal part, which transmits the acquired image to computer and extracts edge of metal part via image processing algorithm, then automatically measures the rectangularity of metal part based on edge template matching. The experimental result shows that this algorithm can automatically detect rectangularity of metal part with certain feasibility.

\section{Composition of detection system}

Figure 1 is the overall structural composition of detection system. This system hardware is composed of workbench and bracket, line camera, lens, illuminant, laser sensor, computer and output equipment, etc ${ }^{[2]}$. The working process is placing rectangle metal part on transparent glass workbench and illuminant under platform, with industrial camera and lens right above the metal part to acquire the image information, which is then transmitted to upper computer via communication interface for storage and analysis.

The image analysis process of the system is mainly developed by Halcon, which is a machine vision integration development software developed by German MVtec and is extensively used in various domains of industrial production. The image processing algorithm herein is divided into four parts: image acquisition, image preprocessing, and template matching and defects detection. For image acquisition, the continuous images captured by camera can be obtained by connecting computer with industrial camera; The image preprocessing part mainly comprises graying, denoising, image 
segmentation and edge extraction, etc. to lower the complexity of later processing; The template matching process comprises obtaining standard image and generating registration template; The defect detection process is to register the preprocessed image against the registration template to detect defects. The entire processing flow is as shown in figure 2 .

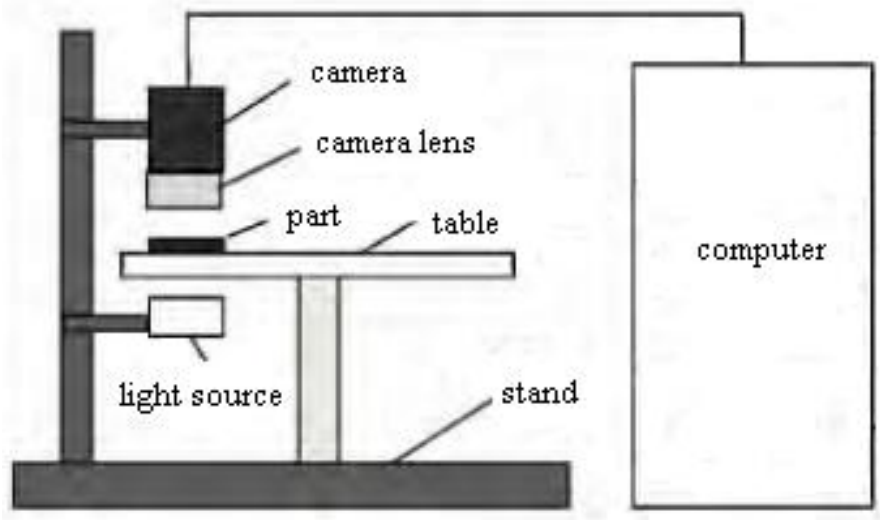

Figure 1. Overall structure of detection system

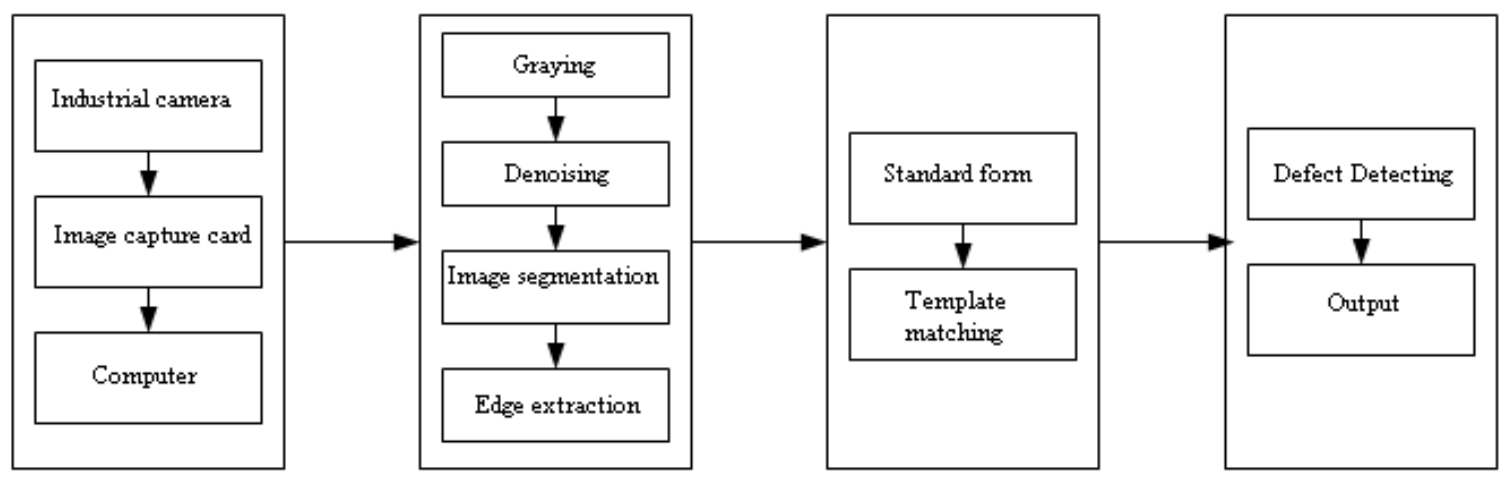

Figure 2. Flow chart for detection system of metal part

\section{Detection Enabling}

\subsection{Image Acquisition}

Image acquisition mainly translates the real image into the digital image which can be processed and stored by computer. The way is connecting computer to industrial camera, and calling open_framegrabber operator in Halcon software to access image acquisition card, then adopting grab_image operator to obtain metal part picture in real time and save it ${ }^{[3]}$.

\subsection{Image Preprocessing}

\subsubsection{Denoise}

The image processed by image acquisition is grayed using rgb1_to_gray operator. These images all contain noise of certain degree. In nature, a lot of noise is in Gaussian distribution, Thus here we adopt Gaussian filter ${ }^{[4]}$ to denoise. The Gaussian filter is obtained by weighting and averaging the value at each pixel for the whole image, here a $3 \times 3$ template is adopted.

\subsubsection{Image Segmentation}

In an effort to partition the target metal part out of background image, the image needs to be binarized. As there is only one target obtained in the process of image acquisition, plus large difference in target and background, the paper adopts threshold segmentation ${ }^{[5]}$, i.e. set the original image as $f(x, y)$, the binarized image as $g(x, y)$, threshold as $T$, then

$$
g(x, y)= \begin{cases}1 & f(x, y)<T \\ 0 & f(x, y) \geq T\end{cases}
$$

Where in the part equal to 1 in binarized image $g(x, y)$ is target metal part, and the otherwise part is background, with statement for segmentation being fast_threshold (Image, Region, 80, 255, 14), 
where Image is input image, Region is segmented image, 80 is the minimal grey scale value for target image, 255 is the maximal grey scale value for target image, and 14 is minimum size of target image.

The inner boundary of metal part for the image segmented using threshold is obtained via boundary operator, then rectangular structural element is used to expand the boundary of target area, lastly the image for segmented metal part area is obtained using reduce_domain operator, with statement used being reduce_domain(Image, EdgeROI,ImageReduced), where Image is original denoised image, EdgeROI is the expanded area boundary, ImageReduced is image for cropped metal part area.

\subsubsection{Edge extraction}

The segmented metal part image has burr of certain degree, which can be eliminated by edge extraction. Here we mainly adopt canny operator ${ }^{[6]}$ to extract edge and obtain the sub-pixel edge contour XLD of target image, with statement used being edges_sub_pix(ImageReduced, Edges, 'canny', 1.7, 40, 120), where ImageReduced is the metal part area after image segmentation, Edges is the XLD contour of image after edge extraction, canny is filter,1.7 is smoothness coefficient, 40 and 120 are respectively lower limit and upper limit of hysteresis threshold.

Extracting XLD of rectangular part is not along the pixels connection point, but the position after interpolation. This entails rectangular fitting for XLD contour of designated length ${ }^{[7]}$, and creating rectangular XLD according fitting parameters, which can improve accuracy of rectangular edge and inhibit pixel value deviating from edge, with operators of fit_rectangle2_contour_xld and gen_rectangle2_contour_xld used for realization.

\subsection{Defect detection}

After obtaining contour XLD of metal rectangular part according to fitting parameters, we can further detect whether the extracted contour of metal part edge is plump, i.e. to detect the rectangularity of metal part. The paper mainly measures rectangularity using the thinking idea of edge template matching ${ }^{[8]}$, with concrete algorithm clue as follows:

(1) Use fitting parameters to generate co-ordinate $(\operatorname{Row} C[i], \mathrm{ColC}[i])$ for four angular points of rectangular part;

(2) Calculate the distance from each point on extracted rectangular contour of edge, set the co-ordinate for any point on the edge contour as $(x, y)$, then the distance from each point to four angular points $D[i]=\operatorname{sqrt}\left((x-\operatorname{Row} C[i])^{2}+(y-\operatorname{Col} C[i])^{2}\right)$, where $i=0,1,2,3$, representing the SN of four angular points ${ }^{[9]}$;

(3) Calculate Dist, the shortest distance from points on edge contour to the four angular points;

(4) Fabricate template, i.e. the corresponding assign is 1 when the distance from the points on edge rectangular contour to angular points is larger than 7 pixels, or assign is 0 , with statement used being: Mask: $=\operatorname{sgn}(\max 2($ Dist-7.0, 0.0) $)$;

(5) Multiply the minimum distance from edge contour point to fitted rectangle and template to solve its maxima and judge whether it is greater than 1, with statement used being Dist_ Mask:= $\max ($ Dist*Mask). If the value is greater than 1, the rectangularity at this pixel is unqualified and edge contour is not plump; if the value is smaller or equal to 1, the rectangularity at this point is qualified, and rectangle is plump ${ }^{[10]}$.

\section{Analysis on detection result}

The experiment first measures 200 specimens using micrometer, including 63 parts with minor defects and the rest qualified ones, then places these specimens in the system to automatic sort them at a speed of 100 parts per minute. The experimental result is as shown in figure 4, wherein (a), (d) and (x) are respectively three metal rectangular parts, (b) , (e)and (y) are respectively contour of extracted edge for the two metal parts, (c), (f)and (z) are respectively the output result after detection of defects. 


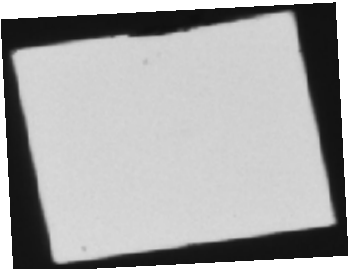

(a)

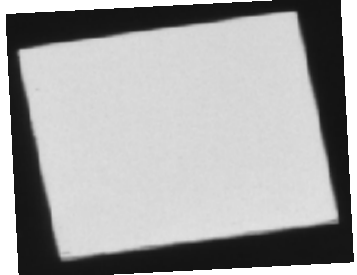

(d)

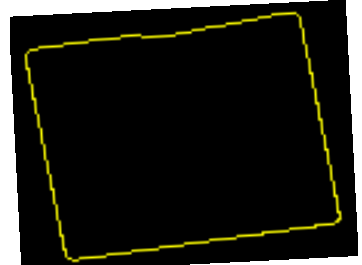

(b)

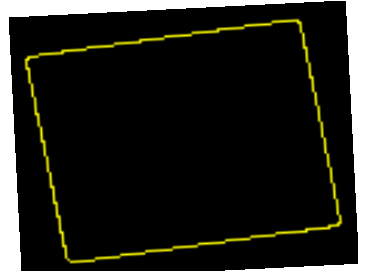

(e)

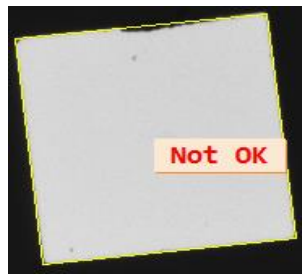

(c)

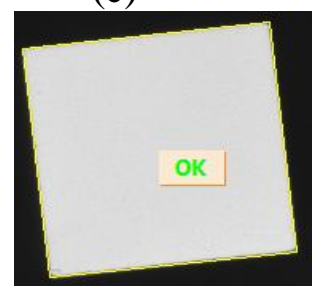

(f)

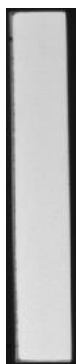

(x)

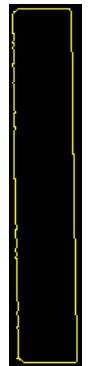

(y)

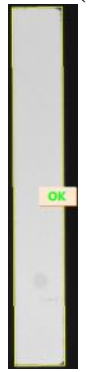

(z)

Figure 3. Comparison of detection effect for rectangularity of metal part

It is observed from the experiment result that this algorithm can reliably detect unqualified rectangular metal part. Although $20 \mathrm{~ms}$ is taken to detect single part, yet as a whole, the result can efficiently and accurately detect rectangularity of metal part.

\section{Conclusion}

The paper suggests a method of detecting rectangularity of metal part based on Halcon, which can automatically select edge contour of metal part to match precisely and effectively detect the non-plump area in image of metal rectangular parts. Compared with traditional artificial detection, this method has marked improvement on speed and accuracy. The experiment result shows that this algorithm can automatically detect the rectangularity of metal part with high feasibility and comprehensive potential application.

\section{Acknowledgements}

The work is supported by the national natural fund project(No.61461023); The project of the education department on Gansu Province(No.2013B-096); The technology project on Lanzhou (No.2014-1-187); The technology project on Lanzhou Institute of Technology (No.15K-009)

\section{References}

[1] Jing Luo, Tingting Dong, Dan Song, Chunb Xiu. The review of surface defect detection[J]. Journal of Frontiers of Computer Science and Technology, 2014, 8(9):1041-1048.

[2] Wencheng Wang. The design of gear defect detection system based on Halcon[J]. Mechanical Transmission, 2014, 38(9):60-63.

[3] Yifeng Chen. The application of 2-dimension detection of workpiece about Halcon[J]. Mechanical and Electrical Technology, 2011(8):12-17. 
[4] Qingli Li, Fan Zhang, Zhongyi Han, Ziqin Li, Tianjie Wang. The study of the Preprocessing algorithm for the images of parts recognition[J]. Combination machine tool and automatic processing technology, 2014,10: 63-65.

[5] Wu Pan, Liyan Zhang, Juncheng Xu. The online detection of workpiece based on machine vision [J]. Combination machine tool and automatic processing technology, 2012(7):75-78.

[6] Pan Yang, Lijun Jang, Zhelin Li. The head crack detection of thread parts based on machine vision [J]. Computer Application and Software, 2013,30(4):51-54.

[7] Xianfeng Zhu, Hongjun Pan. The defects detection and one-dimensional measurement about drum based on Halcon[J]. The JiLin university journal(Information science edition), 2014, 32(3): 308-315.

[8] Xixi Tian, Zhen Kang. A quick template matching method based on image edge[J]. The Journalof Wuhan light industry university, 2014(3):74-78.

[9] Lianglun Chen, Weihuan Jiang. The study of defect detection algorithm in PCB componet installation based on the two-valued projection[J]. Computer Engineering and Design,2010, 31(3): 642-646.

[10]Weifan Wang. The application of the second fusion in the image[J]. Automation and Instrumentation, 2015, 4:182-183.

Introduction on authors:

Xin Liu(1983-), female, Lanzhou, Gansu, lecturer/master, research area:intelligent information processing and image processing; Ying Mu(1982-), female, Tianshui, Gansu, lecturer;Hongbin Wang(1985-), male, Pingliang, Gansu, lecturer/master. 